KANAZAWA-00-12

December, 2000

\title{
Neutrino mass due to the mixing among neutrinos and gauginos
}

\author{
Daijiro Suematsu 固 \\ * Institute for Theoretical Physics, Kanazawa University, \\ Kanazawa 920-1192, JAPAN
}

\begin{abstract}
We consider the explanation of both data of the atmospheric neutrino and the solar neutrino by the neutrino mass matrix derived from the mixing of neutrinos and gauginos in the extended MSSM with an extra U(1) gauge symmetry. This scenario directly relates the neutrino mass to supersymmetry. The structure of the mass hierarchy and the mixing is determined only by the extra $\mathrm{U}(1)$-charge of neutrinos. Although the model is rather simple, it may be able to realize both the small and large mixing angle solutions for the solar neutrino problem. In particular, the large mixing angle MSW solution for the solar neutrino problem can be easily realized. We discuss the relation between the extra $\mathrm{U}(1)$-charge and four solutions for the solar neutrino problem.
\end{abstract}

*e-mail:suematsu@hep.s.kanazawa-u.ac.jp 
The atmospheric and solar neutrino observations have strongly indicated the existence of the neutrino oscillation and then the non-zero neutrino mass [1, 2, 3]. This is an only known evidence suggesting that there is new physics beyond the standard model (SM). Theoretically, the SM has been claimed that there are some unsatisfactory features. Such a representative problem is a gauge hierarchy problem. Supersymmetry has been considered to be a promising candidate to solve it and its various phenomenological features have been studied almost for twenty years 㽤. However, still now there seems not to be an experimental support for it, except for the gauge coupling unification [5]. If we can find that there are some relations between the supersymmetry and the neutrino mass, it is very interesting and might also cause a big impact to the study of the new physics.

In general the supersymmetric model has a special symmetry called $R$-parity defined by $R_{p}=(-1)^{3 B+L+2 S}$, where $B$ is the baryon number, $L$ is the lepton number and $S$ is the spin. The ordinary particles in the SM are $R_{p}$-even and their superpartners are $R_{p}$-odd. As far as the $R$-parity is conserved, neutrinos cannot mix with neutralinos. However, the superpotential can include an $R$-parity violating term like $\epsilon_{\alpha} L_{\alpha} H_{2}$ [6, ], for example, as a result of the gravitational effect. Under the existence of this bi-linear $R$-parity violating term, the vacuum expectation values (VEVs) of sneutrinos is generated and then the mixing among neutrinos and neutralinos is induced. The generation of Majorana neutrino mass in the minimal supersymmetric SM (MSSM) through this mixing has been discussed in a lot of works [8, 6, [7]. In most of them only one of three neutrinos is massive at the tree level [9] and then they could be applied only to the atmospheric neutrino problem [10]. It is necessary to improve this feature in order to explain all of the recent neutrino oscillation data円. One of the possibilities to make other neutrinos massive is to introduce the one-loop effect [12, 13]. In such a framework the solar and atmospheric neutrino problems have been also discussed [13].

In this paper we would like to propose the generation of the small neutrino mass at the tree level based on a typical interaction among neutrinos and gauginos in the usual supersymmetric model. The model is very simple and economical. It needs only a small extension of the MSSM by an extra U(1) gauge symmetry. There are some works [14] on

\footnotetext{
${ }^{1}$ In this paper we confine our study into the explanation of the atmospheric and solar neutrino data. It might be straightforward to extend the model to include a sterile neutrino and make it applicable to the LSND data [11].
} 
the phenomenology including the neutrino mass generation in the supersymmetric model with the spontaneous $R$-parity violation and the extra gauge symmetry. A new feature of our model is that an introduced U(1)-symmetry has generation dependence. The neutrino mass matrix produced in this way has noticeable features for the explanation of both the atmospheric and solar neutrino data. It might realize the large mixing angle solution for them in a natural way if we can assume the spontaneous lepton number violation due to the small VEVs of sneutrinos. Our model could be distinguished at least by the existence of the additional neutral gauge boson $Z^{\prime}$ at the $\mathrm{TeV}$ region from other neutrino models with the large mixing solutions for the solar neutrino problem [15, 16].

In the MSSM there are two neutral gauginos $\lambda_{W_{3}}$ and $\lambda_{Y}$ which are the superpartners of the $\mathrm{SU}(2)_{L}$ and $\mathrm{U}(1)_{Y}$ gauge fields. Their interaction with the ordinary left-handed neutrinos $\nu_{\alpha}(\alpha=e, \mu, \tau)$ can be written as [4]

$$
\frac{i}{\sqrt{2}} g_{2} \sum_{\alpha}\left(\tilde{\nu}_{\alpha}^{*} \lambda_{W_{3}} \nu_{\alpha}-\bar{\lambda}_{W_{3}} \bar{\nu}_{\alpha} \tilde{\nu}_{\alpha}\right)-\frac{i}{\sqrt{2}} g_{1} \sum_{\alpha}\left(\tilde{\nu}_{\alpha}^{*} \lambda_{Y} \nu_{\alpha}-\bar{\lambda}_{Y} \bar{\nu}_{\alpha} \tilde{\nu}_{\alpha}\right) .
$$

If the spontaneous R-parity violation occurs by the VEVs of sneutrinos $\left\langle\tilde{\nu}_{\alpha}\right\rangle \neq 0$, the mixing among gauginos and neutrinos is induced. On the other hand, the supersymmetry breaking causes the gaugino masses $M_{2}$ and $M_{1}$ for $\lambda_{W_{3}}$ and $\lambda_{Y}$ through a suitable mechanism. In the case of $M_{1,2} \gg g_{1,2}\left\langle\tilde{\nu}_{\alpha}\right\rangle$, we can expect to obtain the small Majorana masses of neutrinos as a result of a kind of seesaw mechanism. In fact, if we assume $\left\langle\tilde{\nu}_{e}\right\rangle=\left\langle\tilde{\nu}_{\mu}\right\rangle=\left\langle\tilde{\nu}_{\tau}\right\rangle=u$, the mass mixing among neutrinos and gauginos can be expressed as $\mathcal{L}_{\text {mass }}=-\frac{1}{2}\left(\mathcal{N}^{T} \mathcal{M N}+\right.$ h.c. $)$ and

$$
\mathcal{M}=\left(\begin{array}{ccccc}
0 & 0 & 0 & \frac{g_{2}}{\sqrt{2}} u & \frac{g_{1}}{\sqrt{2}} u \\
0 & 0 & 0 & \frac{g_{2}}{\sqrt{2}} u & \frac{g_{1}}{\sqrt{2}} u \\
0 & 0 & 0 & \frac{g_{2}}{\sqrt{2}} u & \frac{g_{1}}{\sqrt{2}} u \\
\frac{g_{2}}{\sqrt{2}} u & \frac{g_{2}}{\sqrt{2}} u & \frac{g_{2}}{\sqrt{2}} u & M_{2} & 0 \\
\frac{g_{1}}{\sqrt{2}} u & \frac{g_{1}}{\sqrt{2}} u & \frac{g_{1}}{\sqrt{2}} u & 0 & M_{1}
\end{array}\right),
$$

where $\mathcal{N}^{T}=\left(\nu_{\alpha},-i \lambda_{W_{3}}, i \lambda_{Y}\right)$. We consider a special case in which every VEV of sneutrinos is equal in order to reduce the number of parameters and simplify the mass matrix of the model. Under the assumption of $M_{1,2} \gg g_{1,2} u$ we can easily diagonalize the mass matrix $\mathcal{M}$. Although there are three light mass eigenvalues, two of them are zerof?. We must

\footnotetext{
${ }^{2}$ Even if we do not take the VEVs of sneutrinos equal, the situation is the same. In order to improve it the one-loop effect has been taken into account in ref. [7, 13].
} 
extend the MSSM to apply this type of mass generation of neutrinos to the explanation of the atmospheric and solar neutrino data. We consider a model which can naturally change that aspect at the tree level.

Let us consider the introduction of an extra $\mathrm{U}(1)_{X}$ gauge symmetry specified by the following features?. It is non-anomalous and also has flavor diagonal but non-universal couplings. The different charge of $\mathrm{U}(1)_{X}$ is assigned to the fields belonging to the different generationf. It is assumed to be spontaneously broken at a $\mathrm{TeV}$ scale region and then we have an additional neutral gauge bozon $Z^{\prime}$ which is heavier than the ordinary $Z^{0}$. We need a new SM singlet chiral superfield whose scalar component causes the spontaneous breaking of $\mathrm{U}(1)_{X}$ by its VEV. Since a part of the MSSM contents is assumed to have its charge in a generation dependent way, it could generate a non-trivial texture in the mass matrices. However, we cannot use this symmetry for the Froggatt-Nielsen mechanism [19] to induce the hierarchical structure of quark mass matrices since its breaking scale is too small as compared to the Planck scale. For simplicity, we do not assign the $\mathrm{U}(1)_{X}$-charge to quarks and we consider a model in which only leptons have its charges as

$$
\ell_{L} \quad\left(q_{I}, q_{I}, q_{\text {III }}\right), \quad \bar{\ell}_{R} \quad\left(-q_{I},-q_{I},-q_{\text {III }}\right) .
$$

As a result of such a charge assignment $\nu_{\text {III }}$ has a charge $q_{\text {III }}$ different from other neutrinos $\nu_{I}$ and $\nu_{I I}$ whose charge is defined by $q_{I}$ ?. The doublet Higgs superfields are also assumed to have no charge so that the ordinary Yukawa couplings of the charged leptons induce the texture in the mass matrix such as $m_{\text {III }}=m_{\text {IIII }}=0$. Using this charge, the coupling between neutrinos and the $\mathrm{U}(1)_{X}$ gaugino is given by $i \sqrt{2} g_{X} \sum_{\alpha} q_{\alpha}\left(\tilde{\nu}_{\alpha}^{*} \lambda_{X} \nu_{\alpha}-\bar{\lambda}_{X} \bar{\nu}_{\alpha} \tilde{\nu}_{\alpha}\right)$ as eq. (11). We do not consider the kinetic term mixing between the U(1)-gauginos [20]. If we take this effect into account, off-diagonal elements appear in the gaugino mass matrix. In general, the introduction of $\mathrm{U}(1)_{X}$ to the MSSM requires addtional chiral superfields to cancel the gauge anomaly which causes the non-trivial constraint on the charge assignment. Since its breaking scale is in the TeV region, the non-universal couplings of $Z^{\prime}$

\footnotetext{
${ }^{3}$ The additional U(1)-symmetry is known to appear very often in the heterotic superstring models. It can play some useful roles in the supersymmetric models [17].

${ }^{4}$ This kind of charge assignment of $\mathrm{U}(1)_{X}$ has been discussed in the different scenario to explain the small neutrino mass and the proton stability, for example, in [18].

${ }^{5}$ At this stage we cannot determine to which flavor each $\nu_{\alpha}$ corresponds so that we used the Roman numerals for $\alpha$.
} 
with leptons might impose the constraints on the model through the electroweak precision measurements and the flavor changing neutral current (FCNC). We will discuss these points later.

First we focus our attention to the role of $\mathrm{U}(1)_{X}$ in the generation of the neutrino mass due to the mixing among neutrinos and gauginos. Since the gaugino sector is extended, the matrix (2) is modified into f

$$
\mathcal{M}=\left(\begin{array}{cc}
0 & m^{T} \\
m & M
\end{array}\right), \quad m=\left(\begin{array}{ccc}
a_{2} & a_{1} & b \\
a_{2} & a_{1} & b \\
a_{2} & a_{1} & c
\end{array}\right), \quad M=\left(\begin{array}{ccc}
M_{2} & 0 & 0 \\
0 & M_{1} & 0 \\
0 & 0 & M_{X}
\end{array}\right),
$$

where $a_{\ell}=\frac{g_{\ell}}{\sqrt{2}} u, b=\sqrt{2} g_{X} q_{I} u$ and $c=\sqrt{2} g_{X} q_{\text {III }} u$. We can obtain the light neutrino mass matrix from this by using the generalized seesaw formula and it can be written as

$$
M_{\nu}=m^{T} M^{-1} m=\left(\begin{array}{ccc}
m_{0}+\epsilon^{2} & m_{0}+\epsilon^{2} & m_{0}+\epsilon \delta \\
m_{0}+\epsilon^{2} & m_{0}+\epsilon^{2} & m_{0}+\epsilon \delta \\
m_{0}+\epsilon \delta & m_{0}+\epsilon \delta & m_{0}+\delta^{2}
\end{array}\right)
$$

where $m_{0}, \epsilon$ and $\delta$ are defined by

$$
m_{0}=\frac{g_{2}^{2} u^{2}}{2 M_{2}}+\frac{g_{1}^{2} u^{2}}{2 M_{1}}, \quad \epsilon=\frac{g_{X} q_{I} u}{\sqrt{M_{X}}}, \quad \delta=\frac{g_{X} q_{\text {II }} u}{\sqrt{M_{X}}} .
$$

The interesting aspect of this mass matrix is that it is defined only by the gaugino mass $M_{A}(A=\ell, X)$, the gauge couplings $g_{A}$, the $\mathrm{U}(1)_{X}$-charges $q_{\alpha}$ and the VEV $u$ of sneutrinos.

We define the mass eigenstates by $\tilde{\mathcal{N}}_{i}=\left(U^{T}\right)_{i \alpha} \mathcal{N}_{\alpha}$. The diagonalization of the matrix (5) gives

$$
U^{T}=\left(\begin{array}{ccc}
\frac{1}{\sqrt{2}} & -\frac{1}{\sqrt{2}} & 0 \\
\frac{\cos \theta}{\sqrt{2}} & \frac{\cos \theta}{\sqrt{2}} & -\sin \theta \\
\frac{\sin \theta}{\sqrt{2}} & \frac{\sin \theta}{\sqrt{2}} & \cos \theta
\end{array}\right)
$$

where one of the mixing angles $\sin \theta$ is defined as

$$
\sin ^{2} 2 \theta=\frac{8\left(m_{0}+\epsilon \delta\right)^{2}}{\left(m_{0}+2 \epsilon^{2}-\delta^{2}\right)^{2}+8\left(m_{0}+\epsilon \delta\right)^{2}} .
$$

\footnotetext{
${ }^{6}$ In the phenomenological point of view the result obtained in this paper using this mass matrix is independent of the mass generation mechanism. If we can find any models giving the neutrino mass matrix with the features presented here, the result could be also applied to them.
} 


\begin{tabular}{c|ccc}
\hline$(\alpha, \beta)$ & $(i, j)$ & $-4 U_{\alpha i} U_{\beta i} U_{\alpha j} U_{\beta j}(\equiv \mathcal{A})$ & \\
\hline \hline$(I, I I)$ & $(1,2)$ & $\cos ^{2} \theta$ & $(\mathrm{A})$ \\
& $(1,3)$ & $\sin ^{2} \theta$ & $(\mathrm{B})$ \\
& $(2,3)$ & $-\sin ^{2} \theta \cos ^{2} \theta$ & $(\mathrm{C})$ \\
\hline$(I, I I I)$ & $(2,3)$ & $2 \sin ^{2} \theta \cos ^{2} \theta$ & $(\mathrm{D})$ \\
\hline$(I I, I I I)$ & $(2,3)$ & $2 \sin ^{2} \theta \cos ^{2} \theta$ & $(\mathrm{E})$ \\
\hline
\end{tabular}

Table 1. The contributions to each neutrino transition process $\nu_{\alpha} \rightarrow \nu_{\beta}$ from each sector $(i, j)$ of the mass eigenstates.

The non-zero mass eigenvalues are

$$
m_{2,3}=\frac{1}{2}\left\{\left(3 m_{0}+2 \epsilon^{2}+\delta^{2}\right) \mp \sqrt{\left(m_{0}+2 \epsilon^{2}-\delta^{2}\right)^{2}+8\left(m_{0}+\epsilon \delta\right)^{2}}\right\} .
$$

Here we can consistently assume the charged lepton mass matrix is diagonal[ In this case the above mixing matrix $U$ is just the flavor mixing matrix which controls the neutrino oscillation. In the following discussion we assume it in the charged lepton sector. There is no $C P$ violation in the lepton sector under this assumption.

It is well-known that the transition probability due to the neutrino oscillation $\nu_{\alpha} \rightarrow \nu_{\beta}$ after the flight length $L$ is written by using the matrix elements of (7) as

$$
\mathcal{P}_{\nu_{\alpha} \rightarrow \nu_{\beta}}(L)=\delta_{\alpha \beta}-4 \sum_{i>j} U_{\alpha i} U_{\beta i} U_{\alpha j} U_{\beta j} \sin ^{2}\left(\frac{\Delta m_{i j}^{2}}{4 E} L\right)
$$

where $\Delta m_{i j}^{2}=\left|m_{i}^{2}-m_{j}^{2}\right|$. In Table 1 we summalize the contribution to each neutrino transition mode $(\alpha, \beta)$ from each sector $(i, j)$ of the mass eigenstates in our model. As a phenomenologically interesting case, we consider the case in which the mass eigenstates $\tilde{\nu}_{2}$ and $\tilde{\nu}_{3}$ are almost degenerate and the mass hierarchy $m_{1} \ll m_{2} \simeq m_{3}$ is satisfied]. If we use it to explain the atmospheric and solar neutrino data, the difference of the squared

\footnotetext{
${ }^{7}$ Although the $\mathrm{U}(1)_{X}$ constrains the texture of charged leptons mass matrix as mentioned before, some additional symmetry might be necessary to make it diagonal. The large mixing between $\mu$ and $\tau$ is disfavored in this model.

8 This is a well-known reversed hierarchy scenario to the atmosphetic and solar neutrino problems [21]. However, the absolute value of each mass eigenvalue is smaller than the usual scenario because of $m_{1}=0$. Every neutrino cannot be a hot dark matter candidate.
} 
mass should be taken as

$$
\begin{aligned}
& 2 \times 10^{-3} \mathrm{eV}^{2} \approx \Delta m_{12}^{2} \simeq \Delta m_{13}^{2} \approx 6 \times 10^{-3} \mathrm{eV}^{2}, \\
& 10^{-10} \mathrm{eV}^{2} \approx \Delta m_{23}^{2} \sim 1.5 \times 10^{-4} \mathrm{eV}^{2}
\end{aligned}
$$

The suitable value of $\Delta m_{23}^{2}$ should be chosen within the above range depending on which solution is adopted for the solar neutrino problem. We can easily find the simultaneous explanation of both deficit of the atmospheric neutrino and the solar neutrino if we identify the weak eigenstates $(\tau, \mu, e)$ with $(I, I I, I I I)$. Using this identification, eq. (7) is rewritten into the MNS mixing matrix in the usual basis as,

$$
U^{\mathrm{MNS}}=\left(\begin{array}{ccc}
0 & -\sin \theta & \cos \theta \\
-\frac{1}{\sqrt{2}} & \frac{\cos \theta}{\sqrt{2}} & \frac{\sin \theta}{\sqrt{2}} \\
\frac{1}{\sqrt{2}} & \frac{\cos \theta}{\sqrt{2}} & \frac{\sin \theta}{\sqrt{2}}
\end{array}\right) .
$$

The atmospheric neutrino is found to be explained by $\nu_{\mu} \rightarrow \nu_{\tau} \operatorname{composed}$ of (A) and (B) in Table 1. Here we should note that $m_{1}=0$ and also $\Delta m_{12}^{2} \simeq \Delta m_{13}^{2}$ is satisfied. This explanation is independent of the value of $\sin \theta$. On the other hand, the solar neutrino is expected to be explained by $\nu_{e} \rightarrow \nu_{\mu}(\mathrm{E})$ and also $\nu_{e} \rightarrow \nu_{\tau}(\mathrm{D})$. In both processes the amplitude $\mathcal{A}$ is $\frac{1}{2} \sin ^{2} 2 \theta$. If $\sin ^{2} 2 \theta \sim 10^{-2}$, it could realize the MSW small mixing angle solution (SMA) 22. If $\sin ^{2} 2 \theta \sim 1$, it could give the MSW large mixing angle solution (LMA), the low mass MSW solution (LOW) and the vacuum oscillation solution (VO) [22] depending on the value of $\Delta m_{23}^{2}$. The CHOOZ experiment [23] constrains a component $U_{e 1}$ of the MNS mixing matrix in this scenario since the amplitude $\mathcal{A}$ of the contribution to $\nu_{e} \rightarrow \nu_{x}$ with $\Delta m_{12}^{2}$ or $\Delta m_{13}^{2}$ always contains it. This model is free from this constraint since $U_{e 1}=0$.

In Table. 1 an only remaining contribution (C) to $\nu_{\mu} \rightarrow \nu_{\tau}$ cannot imply any evidence in the short baseline experiment even in the case of $\sin ^{2} 2 \theta \simeq 1$ since $\Delta m_{23}^{2}$ is too small. However, this mode might be relevant to the long baseline experiment in the case of $\Delta m_{23}^{2} \sim 10^{-4} \mathrm{eV}^{2}$ which corresponds to the LMA solution of the solar neutrino deficit. We show the effect of the mode $(\mathrm{C})$ on the $P\left(\nu_{\mu} \rightarrow \nu_{x}\right)$ in Fig. 1. The dashed line comes from the modes (A) and (B). This corresponds to the ordinary two flavor oscillation. The thick solid line is the one which is obtained by taking account of the contribution of (C). In the thin solid line the contribution of $(\mathrm{D})$ which corresponds to $\nu_{x}=\nu_{e}$ is also taken into account. This shows that it might be possible to discriminate this model from others 


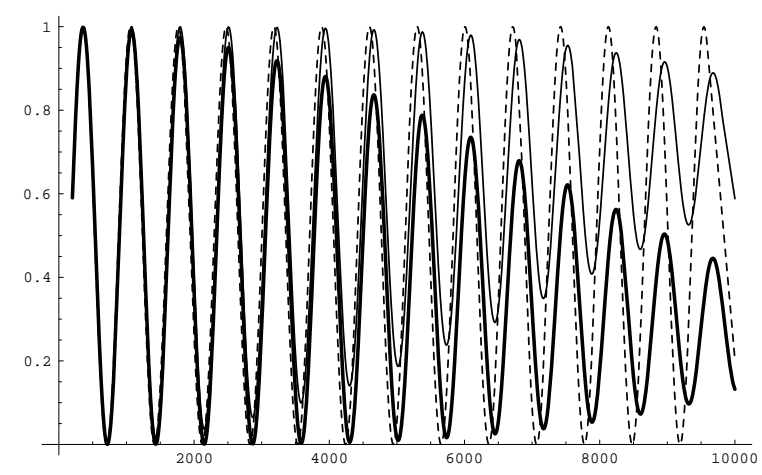

Fig. 1 The transition probability $P\left(\nu_{\mu} \rightarrow \nu_{x(\neq \mu)}\right)$ as a function of the flight length $L$. We assume $E=1 \mathrm{GeV}, \Delta m_{13}^{2}=3.5 \times 10^{-3} \mathrm{eV}^{2}$ and $\Delta m_{23}^{2}=10^{-4} \mathrm{eV}^{2}$

in the long baseline experiment such as $L \underset{\sim}{>} 2000 \mathrm{~km}$. Moreover, this model may be expected to have another experimental signature in the neutrinoless double $\beta$-decay [24]. Using eq. (13), the effective mass parameter which appears in a formula of the rate of neutrinoless double $\beta$-decay is estimated as

$$
\left|m_{e e}\right|=\left.\left|\sum_{j}\right| U_{e j}\right|^{2} e^{i \phi_{j}} m_{j} \mid=\left(m_{2} \sin ^{2} \theta+m_{3} \cos ^{2} \theta\right) \sim m_{3} .
$$

Thus $\left|m_{e e}\right|$ takes the value in the range of $0.04-0.08 \mathrm{eV}$ which is independent of the value of $\sin \theta$, that is, the solution of the solar neutrino problem. The value seems to be within the reach in the near future experiment.

In order to see whether the values of the oscillation parameters can be successfully realized, we need to study them by using the parameters $M_{A}, g_{A}, q_{\alpha}$ and $u$. In the usual soft supersymmetry breaking scenario the gaugino mass is universally produced as $M_{0}$ at the unification scale. Its low energy value is determined by the renormalization group equations (RGEs). If we use the one-loop RGEs, the gaugino mass at a scale $\mu$ can be expressed as

$$
M_{2}(\mu)=\frac{M_{0}}{g_{U}^{2}} g_{2}^{2}(\mu), \quad M_{1}(\mu)=\frac{5}{3} \frac{M_{0}}{g_{U}^{2}} g_{1}^{2}(\mu),
$$

where we assume the gauge coupling unification at the scale $M_{U}$ and define a value of the gauge coupling at $M_{U}$ as $g_{U}$. It is not unnatural to consider the gauge coupling of $\mathrm{U}(1)_{X}$ and its gaugino mass to be the same as the ones of $\mathrm{U}(1)_{Y}$ ? If If we adopt this simplified

\footnotetext{
${ }^{9}$ It might be satisfied if $\mathrm{U}(1)_{X}$ is unified into a simple group together with other SM gauge group like the $\mathrm{SO}(10)$ and $\mathrm{E}_{6}$ models. Also in the superstring context the freedom of an Abelian Kac-Moody level could make it possible.
} 
possibility, we can find that $m_{2,3}$ and $\sin ^{2} 2 \theta$ can be written by using only $q_{\alpha}$ and $\frac{g_{U}^{2}}{M_{0}} u^{2}$ as

$$
\begin{aligned}
& m_{2,3}=\frac{3}{5}\left(2+2 q_{I}^{2}+q_{\text {III }}^{2} \mp \sqrt{\left(2+2 q_{I}^{2}+q_{\text {II }}^{2}\right)^{2}-\frac{16}{3}\left(q_{I}-q_{\text {III }}\right)^{2}}\right) \frac{g_{U}^{2} u^{2}}{M_{0}}, \\
& \sin ^{2} 2 \theta=\frac{8\left(2+3 q_{I} q_{\text {III }}\right)^{2}}{\left(2+6 q_{I}^{2}-3 q_{\text {II }}^{2}\right)^{2}+8\left(2+3 q_{I} q_{\text {III }}\right)^{2}} .
\end{aligned}
$$

The structure of the mass spectrum and the flavor mixing is controled by the $\mathrm{U}(1)_{X^{-}}$ charge. The gaugino mass $M_{0}$ and the VEV $u$ of sneutrinos are relevant to the mass eigenvalues only in the form of an overall factor $\frac{g_{U}^{2}}{M_{0}} u^{2}$. In order to realize the value of (11) which is required by the atmospheric neutrino deficit, $\frac{g_{U}^{2}}{M_{0}} u^{2}$ needs to be in the range of $0.017 \mathrm{eV} \lesssim \frac{g_{U}^{2}}{M_{0}} u^{2} \lesssim 0.023 \mathrm{eV}$. If we take $M_{0} \sim 100 \mathrm{GeV}$ and $g_{U} \sim 0.72$, for example, it shows that the sneutrino VEV should be $u \sim 60-70 \mathrm{keV}$. The remaining freedom which we can use to explain the solar neutrino deficit is the $\mathrm{U}(1)_{X}$-charge of neutrinos. In Fig. 2 we plot the value of the $\mathrm{U}(1)_{X^{-}}$-charge and the corresponding oscillation parameters which are suitable for the explanation of the solar neutrino deficit. The figure shows that the reasonable value of the $\mathrm{U}(1)_{X}$-charge can realize all the well-known solutions for the solar neutrino problem. As is easily seen from eq. (16), $\Delta m_{23}^{2}$ is propotional to the part of square root in the expression of $m_{2,3}$. It means that the solution with the smaller

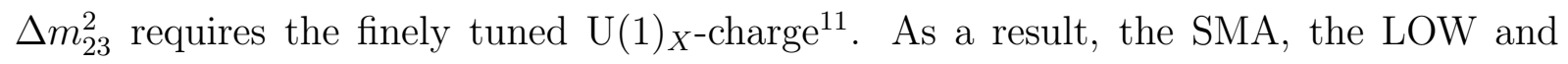
the VO seem to require the finer tuning of the $\mathrm{U}(1)_{X}$-charge than the LMA. It might be favorable for this model if we take seriously the recent Super-Kamiokande analysis of the solar neutrino [3]. Anyway it is interesting that every solution is obtainable within this simplified framework.

Now we comment on some remaining important points. The first point is whether the anomaly-free $\mathrm{U}(1)_{X}$ can realize the solutions in Fig. 2(a). We show it by using a simple example presented in (3). In order to cancel the gauge anomaly we introduce additional chiral superfields with the non-zero $U(1)_{X}$-charge: $\mathbf{2}_{0}, \mathbf{1}_{ \pm 1}$ and $4\left(\mathbf{1}_{0}\right)$, where we indicate the representation under $\mathrm{SU}(2)_{L} \times \mathrm{U}(1)_{Y}$. The $\mathrm{U}(1)_{X}$-symmetry is assumed to be broken by the VEVs of $\mathbf{1}_{0}$ 's. The $\mathbf{2}_{0}$ and $\mathbf{1}_{ \pm 1}$ should be massive at the TeV scale through the VEVs. If we impose this requirement with the anomaly-free conditions on the $\mathrm{U}(1)_{X^{-}}$

\footnotetext{
${ }^{10}$ In the estimation we have already taken account of the effect coming from the $\mathrm{U}(1)_{X^{-}}$-charge dependence. However, its effect is not large and only a factor of $O(1)$.

${ }^{11}$ It may be useful to note that the complete degeneracy $m_{2}=m_{3}$ occurs in the case of $q_{I}=1 / \sqrt{3}$ and $q_{\text {III }}=-2 / \sqrt{3}$, which satisfy $\sum_{\alpha} q_{\alpha}=0$.
} 
(a)

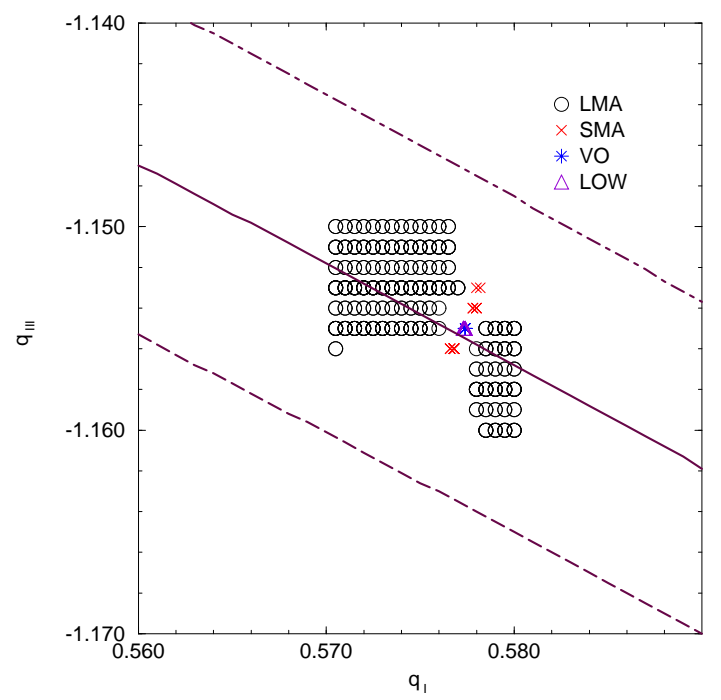

(b)

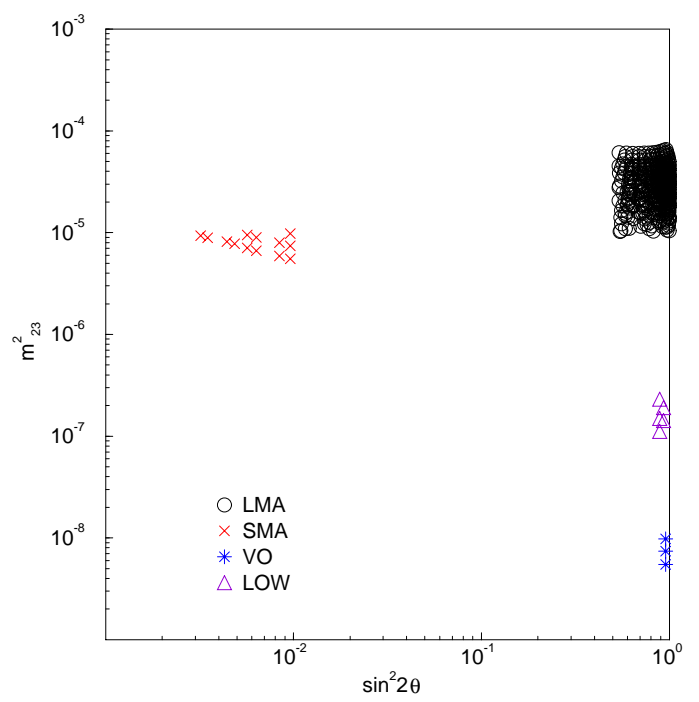

Fig. 2 (a) Scatter plots of the $\mathrm{U}(1)_{X}$-charge of neutrinos which can give the solution to the atmospheric and solar neutrino problems. The dot-dashed, solid and dashed lines are the ones corresponding to $Q=1.04,1.05$ and 1.06, respectively. (b) Oscillation parameters for the solar neutrino which are realized by the $\mathrm{U}(1)_{X}$-charge of neutrinos shown in (a).

charges, we find that the charges of all fields can be expressed by using the charge $Q$ of one of $\mathbf{1}_{0}$ 's. In Fig. 2(a) we plot the values of $\left(q_{I}, q_{\text {III }}\right)$ of such solutions by taking suitable values of $Q$. From it we find that our scenario could have the solutions consistent with the above mentioned conditions. Although we take a very simple example to show it, it would be possible to construct more elaborate examples.

The next point is the phenomenological constraint on the $\mathrm{U}(1)_{X}$ since we have a $Z^{\prime}$ at a scale not far from the weak scale. The constraint on the $Z^{\prime}$ comes from the direct search [25] and the electroweak precision measurements [26, 17]. In general these seem to require the mass bound $m_{Z^{\prime}}>600 \mathrm{GeV}$, which can be consistent with our assumption of the $\mathrm{U}(1)_{X}$ breaking scale. Another constraint could also come from the FCNC in the lepton sector such as the coherent $\mu$-e conversion, $\tau \rightarrow 3 e, 3 \mu$ and $\mu \rightarrow$ er etc., since we assume the flavor diagonal but non-universal couplings of $Z^{\prime}$ to leptons. The detailed analysis for such a issue has been done in [27] and we can use the discussion there. In our model the $Z^{\prime}$ interaction term can be written in the mass eigenstates as,

$$
\mathcal{L}_{Z^{\prime}}=-g_{1}\left[\frac{g_{X}}{g_{1}} \cos \xi J_{(X)}^{\mu}-\sin \xi J_{(1)}^{\mu}\right] Z_{\mu}^{\prime},
$$




$$
\begin{aligned}
& J_{(X)}^{\mu}=\sum_{i j}\left[\bar{\nu}_{L_{i}} B_{i j}^{\nu_{L}} \nu_{L_{j}}+\bar{\ell}_{L_{i}} B_{i j}^{\ell_{L}} \ell_{L_{j}}+\bar{\ell}_{R_{i}} B_{i j}^{\ell_{R}} \ell_{R_{j}}\right], \\
& B_{i j}^{\psi}=V^{\psi \dagger} \operatorname{diag}\left(q_{\text {III }}, q_{I}, q_{I}\right) V^{\psi},
\end{aligned}
$$

where $\xi$ is a $Z-Z^{\prime}$ mixing angle and $V^{\psi}$ is the unitary matrix to diagonalize the mass matrix of $\psi$. The FCNC in the lepton sector causes the strong constraint on $B_{i j}^{\ell_{L, R}}$ as it is discussed in [27]. Since the $\mathrm{U}(1)_{X^{-}}$charge of $\mu$ and $\tau$ is equal in our model, $B_{23}^{\ell_{L}, R}=0$. Moreover, if we remind that $m_{e \mu}=m_{e \tau}=0$ is satisfied in the charged lepton mass matrix because of the constraint of the $\mathrm{U}(1)_{X}$ charge, we find that $B_{12}^{\ell_{L}, R}=B_{13}^{\ell_{L, R}}=0$. Then the non-universal couplings of $Z^{\prime}$ do not induce the observable effect on the FCNC. The addtional contribution to the FCNC could be caused by the extended neutralino sector. The study in that case has been done in [20] for the models which can be embedded in $\mathrm{E}_{6}$. The effect is found not to be so large as far as we take parameters in such a way that the MSSM satisfies the experimental bound. Although the $\mathrm{U}(1)_{X}$-symmetry is different from this case, the qualitative feature is similar also in the present model. Thus as far as we consider a TeV scale $Z^{\prime}$ with the small mixing $\xi \lesssim 10^{-3}$, the constraint from the precision measurement and the FCNC of the lepton sector seems not severe to the present model.

Another important point is the origin of the small VEV $u$ of sneutrinos. As mentioned in the previous part, it should be around $O\left(10^{2}\right) \mathrm{keV}$ which is much smaller than the weak scale. In the MSSM there are arguements on the lepton number violation due to the VEVs of sneutrinos in the vicinity of the weak scale [28] and also some authors point out that the neutrino mass produced by them can be sufficiently small [13, 7]. However, in our scenario we need much smaller VEVs of sneutrinos than the weak scale. As one of the possibilities, we might consider that such small VEVs of sneutrinos could be obtained if there is bi-linear $R$-parity violating terms $\epsilon L_{\alpha} H_{2}$ with the sufficiently small $\epsilon$ ए2. We can check it briefly by minimizing the scalar potential under the assumption that $\left\langle H_{1,2}\right\rangle$ can be treated as constants. In this case the value of $u$ derived from the potential minimization condition can be approximately written as

$$
u \sim \frac{6 \epsilon\left(\mu\left\langle H_{1}\right\rangle+B_{\epsilon}\left\langle H_{2}\right\rangle\right)}{3\left(g_{1}^{2}+g_{2}^{2}\right)\left(\left\langle H_{1}\right\rangle^{2}-\left\langle H_{2}\right\rangle^{2}\right)+2 g_{X}^{2}\left(\sum_{\alpha} q_{\alpha}\right)\left(q_{1}\left\langle H_{1}\right\rangle^{2}+q_{2}\left\langle H_{2}\right\rangle^{2}\right)+6 m^{2}},
$$

where $B_{\epsilon}$ is a soft supersymmetry breaking parameter related to the $\epsilon L_{\alpha} H_{2}$ terms and

\footnotetext{
${ }^{12}$ We assume $\epsilon_{i}=\epsilon$. The smallness of $\epsilon$ also causes a new hierarchy problem. To resolve this problem we might be able to use the similar mechanism to the solutions for the $\mu$ problem [29], although the relevant energy scale needs to be scaled down by some orders of magnitude.
} 
$m^{2}$ is the soft scalar mass of sneutrinos, which is assumed to be universal. From this we find that the sufficiently small $u$ can be obtained as far as $\epsilon$ is small enough and the $\mu$-parameter, $B_{\epsilon}$ and $m^{2}$ take the values of the order of weak scale. However, the $\epsilon L_{\alpha} H_{2}$ terms generate the $\nu_{\alpha}-\tilde{H}_{2}^{0}$ mixing which affects our analysis. In order to escape the influence due to the $\epsilon$ terms, $\frac{g_{A} u}{M_{A}}$ might be required to be sufficiently larger than $\frac{\epsilon}{\mu}$. We need to check whether these conditions are satisfied at the true vacuum with the equal VEVs of sneutrinos taking account of the radiative correction. Including the study of other possibility to realize the small $u$, we would like to leave it in the future publication instead of going further into it here.

In summary we have proposed the scenario for the mass and the mixing of neutrinos in the supersymmetric model with an extra $U(1)_{X}$-symmetry. The scenario is based on the mixing among neutrinos and gauginos. In this model we could obtain the non-zero mass eigenvalues for neutrinos at the tree level. The atmospheric and solar neutrino deficits can be simultaneously explained by the reversed mass hierarchy scenario. In particular, every known solution for the solar neutrino problem can be realized only by tuning the $\mathrm{U}(1)_{X}$-charge of neutrinos. It is interesting that the large mixing angle MSW solution can be easily realized as compared to other solutions. The neutrinoless double $\beta$-decay might be accessible if the experimental bound is improved to the level of $\left|m_{e e}\right| \sim 0.04$ $0.08 \mathrm{eV}$. There remain some unsolved problems related to the VEVs of sneutrinos and the supersymmetry breaking parameters. Further investigation of these problems seems to be necessary to see whether our model works well in a realistic way. Finally we would like to stress that the texture of the present mass matrix might be scaled up into the high energy region. In that case it may be regarded as the usual seesaw model with a

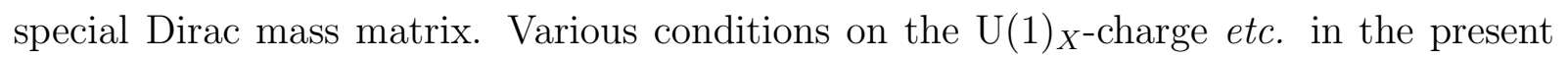
scenario could be replaced into the ones for Yukawa couplings and so on. The study in this direction also seems to be interesting.

I would like to thank M. Tanimoto for useful comments related to the CHOOZ constraint. This work is supported in part by the Grant-in-Aid for Scientific Research from the Ministry of Education, Science and Culture (No.11640267). 


\section{References}

[1] Y. Fukuda et at., Phys. Rev. Lett. 81 (1998) 1562; R. Becker-Szendy et al. IMB collaboration, Nucl. Phys. B38 (proc. suppl.) (1995) 331; W. W. M. Allison et al., Soudan collaboration, Phys. Lett. B391 (1997) 491; M. Ambrosio et al., Phys. Lett. B434 (1998) 451.

[2] B. T. Cleveland et al., Ap. J. 496 (1998) 505; K. S. Hirata et al., Kamiokande collaboration, Phys. Rev. Lett. 77 (1996) 1683; W. Hampel, GALLEX collaboration, Phys. Lett. B388 (1996) 384; J. N. Abdurashitov et al., SAGE collaboration, Phys. Rev. Lett. 77 (1996) 4708.

[3] Y. Suzuki, Super-Kamiokande collaboration, Talk presented at Neutrino 2000, Sudbury, Canada.

[4] H.-P. Nilles, Phys. Rep. C110 (1984) 1; H. E. Haber and G. I. Kane, Phys. Rep. C117 (1985) 75.

[5] U. Amaldi, W. de Boer and H. Furstenau, Phys. Lett. B260 (1991) 447; J. Ellis, S. Kelley and D. V. Nanopoulos, Phys. Lett. B260 (1991) 131; P. Langacker and L. Luo, Phys. Rev. D44 (1991) 817; C. Giunti, C. W. Kim and U. W. Lee, Mod. Phys. Lett. A6 (1991) 1745.

[6] F. de Compos, M. A. Garcá-Jareño, A. S. Joshipura, J. Rosiek and J. W. F. Valle, Nucl. Phys. B451 (1995) 3; T. Banks, Y. Grossman, E. Nardi and Y. Nir, Phys. Rev. D52 (1995) 5319; A. S. Joshipura and M. Nowakowshi, Phys. Rev. D51 (1995) 2421.

[7] M. A. Díaz, J. C. Romão and J. W. F. Valle, Nucl. Phys. B524 (1998) 23; J. W. F. Valle, hep-ph/9808292 and references therein.

[8] G. G. Ross and J. W. F. Valle, Phys. Lett. B151 (1985) 375; J. Ellis, G. Gelmini, C. Jarlskog, G. G. Ross and J. W. F. Valle, Phys. Lett. B150 (1985) 142; C. S. Aulakh and R. N. Mohapatra, Phys. Lett. B121 (1983) 14.

[9] A. Santamaria and J. W. F. Valle, Phys. Lett. B195 (1987) 423; Phys. Rev. D39 (1989) 1780; Phys. Rev. Lett. 60 (1988) 397. 
[10] V. Bednyakov, A. Faessler and S. Kovalenko, Phys. Lett. B442 (1998) 203.

[11] C. Athanassopoulos et al., Phys. Rev. Lett. 77 (1996) 3082.

[12] R. Hempfling, Nucl. Phys. B478 (1996) 3; E. Nardi, Phys. Rev. D55 (1997) 5772; A. S. Joshipura and S. K. Vempati, hep-ph/9903435; D. E. Kaplan and A. E. Nelson, hep-ph/9901254.

[13] J. C. Romão, M. A.Díaz, M. Hirsch, W. Porod and J. W. F. Valle, Phys. Rev. D61 (2000) 071703; M. Hirsch, M. A.Díaz, W. Porod, J. C. Romão and J. W. F. Valle, hep-ph/0004115.

[14] J. W. F. Valle, Phys. Lett. B196 (1987) 157; M. C. Gonzalez-Garcia and J. W. F. Valle, Nucl. Phys. B355 (1991); K. Huitu and J. Maalampi, Phys. Lett. B344 (1995) 217.

[15] V. Barger, S. Pakvasa, T. J. Weiler and K. Whisnant, Phys. Lett. B437 (1998) 107; S. Davidson and S. F. King, Phys. Lett. B445 (1998) 191; R. N. Mohapatra and S. Nussinov, Phys. Rev. D60 (1999) 013002.

[16] For the recent models with the large mixing, see for example, J. M. Mira, E. Nardi, D. A. Restrepo and J. W. F. Valle, Phys. Lett. 492 (2000) 81; A. de Gouvea and J. W. F. Valle, hep-ph/0010299; P. H. Chankowski, A. Ioannisian, S. Pokorski and J. W. F. Valle, hep-ph/0011150; Q. Shafi and Z. Tavartkiladze, hep-ph/0101350; S. F. King and M. Oliveira, hep-ph/0009287.

[17] D. Suematsu and Y. Yamagishi, Int. J. Mod. Phys. A10 (1995) 4521; M. Cvetič and P. Langacker, Phys. Rev. D54 (1996), Mod. Phys. Lett. A11 (1996) 1247; D. Suematsu, Phys. Rev. D59 (1999) 055017.

[18] E. Nardi, Phys. Rev. D48 (1993) 3277; Phys. Rev. D49 (1994) 4394; E. Nardi and T. Rizzo, Phys. Rev. D50 (1994) 203; D. Suematsu, Prog. Theor. Phys. 96 (1996) 611.

[19] C. Froggatt and H. B. Nielsen, Phys. Lett. B147 (1979) 277.

[20] D. Suematsu, Mod. Phys. Lett. A12 (1997) 1709; Phys. Lett. B416 (1998) 108; Phys. Rev. D57 (1998) 1738. 
[21] S. T. Petcov and A. Yu. Smirnov, Phys. Lett. B322 (1994) 109; D. O. Caldwell and R. N. Mohapatra, Phys. Lett. B354 (1995) 371; G. Rafflet and J. Silk, Phys. Lett. B366 (1996) 429.

[22] J. N. Bahcall, P. I. Krastev, A. Yu. Smirnov, hep-ph/0006078; M. C. GonzalezGarcia, P. C. de Holanda, C. Pena-Garay, J. W. F. Valle, hep-ph/9906469.

[23] C. Bemporad, CHOOZ collaboration, Nucl. Phys. B (Proc. Suppl.) 77 (1999) 159.

[24] S. M. Bilenky, C. Giunti, C. W. Kim and S. T. Petcov, Phys. Rev. D54 (1996) 4432; S. M. Bilenky, C. Giunti, W. Grimus, B. Kayser and S. T. Petcov, Phys. Lett. B465 (1999) 193; H. V. Klapdor-Kleingrothaus, H. Päs and A. Yu. Smirnov, hep-ph/0003219.

[25] F. Abe, et al., CDF collaboration, Phys. Rev. Lett. 79 (1997) 2192.

[26] For example, J. Erler and P. Langacker, Phys. Lett B456 (1999) 68; G. C. Cho, K. Hagiwara and Y. Umeda, Nucl. Phys. B531 (1998) 65, Nucl. Phys. B555 (1999) 651 (E); and references therein.

[27] P. Langacker and M. Plumacher, Phys. Rev. D62 (2000) 013006.

[28] A. Masiero and J. W. F. Valle, Phys. Lett. B251 (1990) 273; J. C. Romão, C. A. Santos and J. W. F. Valle, Phys. Lett. B288 (1992) 311; J. C. Romão, A. Ioannisian and J. W. F. Valle, Phys. Rev. D55 (1997) 427.

[29] For a recent review, see for example, N. Polonsky, hep-ph/9911329. 